\section{DNMT1 represses $p 53$ to maintain progenitor cell survival during pancreatic organogenesis}

\author{
Senta Georgia, ${ }^{1,3,4}$ Murtaza Kanji, ${ }^{1}$ \\ and Anil Bhushan ${ }^{1,2}$ \\ ${ }^{1}$ Department of Medicine, University of California \\ at Los Angeles, Los Angeles, California 90024, USA; ${ }^{2}$ Molecular, \\ Cell, and Developmental Biology, University of California \\ at Los Angeles, Los Angeles, California 90095, USA
}

In the developing pancreas, self-renewal of progenitors and patterning of cell fates are coordinated to ensure the correct size and cellular makeup of the organ. How this coordination is achieved, however, is not clear. We report that deletion of DNA methyltransferase 1 (Dnmt1) in pancreatic progenitors results in agenesis of the pancreas due to apoptosis of progenitor cells. We show that DNMT1 is bound to the $p 53$ regulatory region and that loss of Dnmt1 results in derepression of the p53 locus. Haploinsufficiency of $p 53$ rescues progenitor cell survival and cellular makeup of the Dnmt1-deleted pancreas.

Supplemental material is available for this article.

Received September 27, 2012; revised version accepted January 14, 2013.

The vertebrate pancreas is a complex organ made of functionally different tissue types that arise from a common progenitor cell pool during embryogenesis (Gu et al. 2003; Jensen 2004; Gittes 2009). Lineage tracing analysis indicates that a pool of pancreatic progenitor cells persists throughout organogenesis to give rise to differentiated mature cell types in a spatially and temporally regulated manner (Herrera 2000; Jensen et al. 2000a; Gu et al. 2002). The maintenance of the progenitor cell pool requires that progenitor cell self-renewal is balanced with cell cycle exit to coordinate differentiation of mature cell types of the pancreas (Georgia et al. 2006).

DNA methylation is a potential epigenetic mechanism that may be involved in maintaining the balance between self-renewal and terminal differentiation in the developing pancreas. DNA methylation may serve as a means of repressing pluripotency genes in multipotent progenitor cells or a means of repressing lineage-specific differentiation genes in multipotent progenitor cells (Reik 2007). It is not clear whether pancreatic progenitor DNA methylation patterns must be inherited during self-renewal to maintain a functional progenitor population by repres-

[Keywords: pancreatic development; DNMT1; differentiation; DNA methylation; p53]

${ }^{3}$ Present address: Division of Endocrinology, Children's Hospital Los Angeles, 4650 Sunset Blvd., Los Angeles, CA 90027, USA.

${ }^{4}$ Corresponding author

E-mail sgeorgia@chla.usc.edu

Article is online at http://www.genesdev.org/cgi/doi/10.1101/gad.207001.112. sing differentiation genes or to serve as a basis for establishing lineage-specific methlylation patterns during differentiation.

DNA methyltransferase 1 (Dnmt1) is the enzyme that propagates DNA methylation patterns during cell division. Previous studies on Dnmt1 have yielded interesting yet divergent data about its role in maintenance and differentiation of progenitor cell types. Dnmt1-null mouse embryonic stem cells (mESCs) undergo limited differentiation and apoptosis and are eliminated in competition assays with wild-type mESCs yet have a proliferative capacity comparable with that of wild-type mESCs when maintained in an undifferentiated state (Lei et al. 1996; Panning and Jaenisch 1996). Dnmt1-null embryos expire from growth arrest and severe neural tube distortions by embryonic day 8.5 (E8.5), which indicates that Dnmt1-null mESCs are capable of undergoing more extensive differentiation in vivo than in vitro ( $\mathrm{Li}$ et al. 1992; Lei et al. 1996). Loss of Dnmt1 in hematopoetic progenitor cells results in defects in self-renewal and niche retention and an inappropriate shift to myeloid progenitor cell cycling and differentiation (Trowbridge et al. 2009). Studies in human adult epidermal stem cells indicate that Dnmt1 maintains progenitor self-renewal by repressing genes that trigger cell cycle exit and premature differentiation (Sen et al. 2010). During neurogenesis, Dnmt1 restricts precocious astrogliogenesis of neural precursor cells, but deletion of Dnmt1 in telencephalic precursors results in cell death and forebrain degeneration (Fan et al. 2005; Hutnick et al. 2009). Taken together, these studies present context-dependent roles for Dnmt1 that may have differential effects in vitro or in vivo.

To investigate the in vivo role of DNMT1 in progenitor maintenance and concomitant lineage specification during organogenesis, we conditionally deleted Dnmt1 in pancreatic progenitor cells. Analysis of progenitor cell self-renewal dynamics revealed that Dnmt1-null pancreatic progenitor cells undergo G2 arrest. Our studies indicate that DNMT1 functions to repress $p 53$. In its absence, p53 expression is derepressed, resulting in apoptosis of pancreatic progenitor cells. We show that p53 haploinsufficiency rescues progenitor cell survival and restores pancreatic organogenesis in the Dnmt1-null embryos. These data suggest that Dnmt1 is required to prevent p53-dependent apoptosis during lineage specification of pancreatic progenitor cells.

\section{Results and Discussion}

\section{Loss of Dnmt1 results in a severely atrophic pancreas}

We bred mice with a floxed allele of Dnmt1 (DNMT1 ${ }^{\mathrm{fl} / \mathrm{fl}}$ ) with mice transgenic for Cre recombinase under the control of the Pdx1 promoter (Jackson-Grusby et al. 2001; Gu et al. 2002). This restricted Dnmt1 excision to the pancreatic epithelium (DNMT1 ${ }^{\mathrm{PC}}$ ). To facilitate lineage tracing studies, we also bred in the stop-floxed$\mathrm{R} 26 \mathrm{R}^{\mathrm{YFP}}$ to mark all cells derived from progenitor cells that expressed the Pdx1-Cre transgene (Srinivas et al. 2001). To confirm that deletion of Dnmt1 resulted in hypomethylation of the self-renewing pancreatic progenitor pool, we used immunohistochemistry to detect 5-methyl-cytosine $(5 \mathrm{mC})$. Unlike the control epithelium, 
where $5 \mathrm{mC}$ uniformly stained the epithelium and surrounding mesenchyme, $5 \mathrm{mC}$ staining was grossly diminished in the epithelium of the DNMT1 ${ }^{\mathrm{PC}}$ pancreas but maintained in the surrounding mesenchyme (Supplemental Fig. 1A,B). This indicated that deletion of Dnmt1 resulted in hypomethylation of the pancreatic epithelium during organogenesis. Previous reports have shown that loss of Dnmt1 leads to derepression of intracisternal A particle (IAP), a core retroviral element protein (Fan et al. 2001). Consistent with these previous studies, IAP staining was not detected in the pancreatic epithelium of control E13.5 embryos, but high levels of IAP were detected in the DNMT1 ${ }^{\mathrm{PC}}$ pancreatic epithelium (Supplemental Fig. 1C,D). Some glucagon-positive cells in the DNMT1 ${ }^{\mathrm{PC}}$ pancreatic epithelium were negative for IAP expression and were likely derived from cells that had escaped Cremediated recombination. These data indicated that $P d \times 1$ Cre expression efficiently deleted Dnmt1 from the large majority of pancreatic progenitor cells early in development and led to hypomethylation of the pancreatic epithelial cells.

We next examined the effects of Dnmt1 deletion on pancreatic organogenesis by analyzing DNMT1 ${ }^{\mathrm{PC}}$ litters at birth. DNMT1 ${ }^{\mathrm{PC}}$ animals were born alive at expected Mendelian ratios. Examination of littermates indicated that the pancreas of pups in which one allele of Dnmt1 was deleted were grossly normal and comparable with wild-type control littermates (Fig. 1 A,B, D,E). YFP expression was absent in the control animals and homogenously distributed throughout the pancreas in heterozygous pups (Fig. 1G,H). Strikingly, gross examination of the DNMT1 ${ }^{\mathrm{PC}}$ pancreas revealed a severely atrophic pancreas (Fig. 1C,F). The rudimentary DNMT1 ${ }^{\text {PC }}$ pancreas displayed little YFP expression, indicating that most of the atrophic pancreatic tissue was derived from cells that had escaped recombination (Fig. 1I). These data

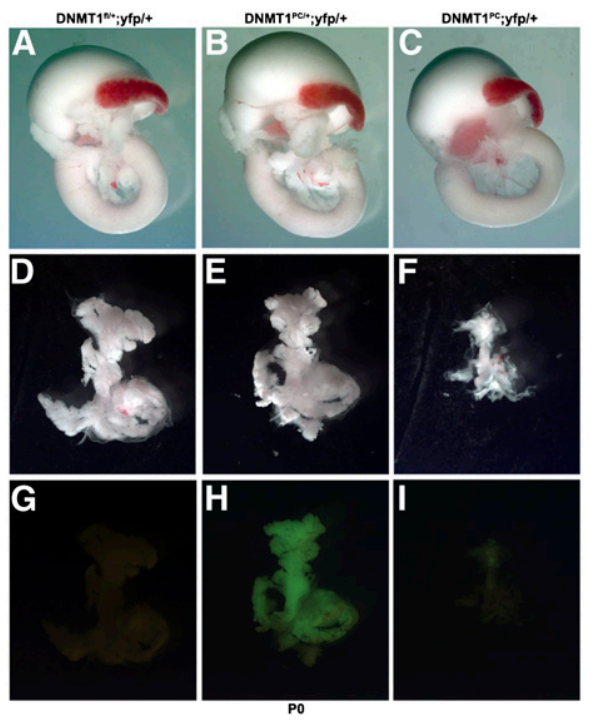

Figure 1. DNMT1 deletion results in an atrophic pancreas. $(A-C)$ Gastrointestinal tract of control (DNMT1 $\left.1^{\mathrm{fl} / \mathrm{fl}}\right)(A)$, heterozygous (DNMT1 ${ }^{\mathrm{PC} /+}$ ) $(B)$, and conditional knockout $\left(\mathrm{DNMT} 1^{\mathrm{PC}}\right)(C)$ pancreas at postnatal day $0(\mathrm{P} 0)$. $(D-F)$ Pancreas dissected from the gastrointestinal tract illustrate the atrophic size of the DNMT1 ${ }^{\text {PC }}$ pancreas. $(G-I)$ Lineage trace analysis indicates that there is efficient recombination in the heterozygous DNMT1 $1^{\mathrm{PC} /+}$ pancreas $(H)$, but very few recombined cells persist in the DNMT1 ${ }^{\mathrm{PC}}$ pancreas $(I)$. indicated that Dnmt1 is essential for the formation of the pancreas.

Previous studies in zebrafish indicated that loss of Dnmt1 resulted in degeneration of the acinar pancreas, but ductal and endocrine lineages were spared, leaving open the possibility that the atrophic pancreas that we observed could consist of primarily endocrine and ductal cells (Anderson et al. 2009). To determine whether the absence of Dnmt1 resulted in loss of specific cell lineages, we carried out immunohistological analysis for differentiated cell types in the DNMT1 ${ }^{\mathrm{PC}}$ pancreas. Antibody staining against exocrine cells expressing amylase and endocrine cells expressing insulin showed that scattered clusters of both endocrine and exocrine cells were present in the DNMT1 ${ }^{\mathrm{PC}}$ pancreas, but the typical rosette architecture of the acinar tissue and islet clusters of insulin cells was disrupted (Supplemental Fig. S2A-D). Cells that stained for the ductal marker mucin were scattered throughout the DNMT1 ${ }^{\mathrm{PC}}$ pancreas (data not shown). From this analysis, we concluded that deletion of Dnmt1 from pancreatic epithelial progenitor cells did not impact any particular lineage and instead disrupted the architecture and severely reduced the numbers of differentiated cells of all lineage of the mature pancreas.

\section{Deletion of Dnmt1 in pancreatic progenitors results in the absence of differentiated cells}

We investigated whether a severely reduced pancreas size could result from depletion of the pancreatic progenitor pool during embryogenesis. We examined DNMT1 ${ }^{\text {PC }}$ and control littermate pancreas by immunostaining for pancreatic progenitor markers Pdx1 and Sox9. At E13.5, the overlapping Pdx1 and Sox9 populations were comparable in the control and DNMT1 ${ }^{\mathrm{PC}}$ pancreatic epithelium $(P=0.2568)$ (Fig. 2A,B,E). In contrast, the number of cells staining for Sox 9 at E15.5 was reduced by $>40 \%$ in the DNMT $1^{\text {PC }}$ pancreas compared with the control littermate pancreas $(P=0.0137)$ (Fig. $2 \mathrm{C}, \mathrm{D}, \mathrm{E})$. This indicated that the progenitor pool is depleted during pancreatic organogenesis and results in a severely atrophic pancreas. The depletion of pancreatic progenitors could be due to either a defect in self-renewal or precocious differentiation. Precocious differentiation leading to depletion of the pancreatic progenitor pool has been previously described (Jensen et al. 2000b; Bhushan et al. 2001). To assess precocious differentiation, we carried out immunohistochemistry of E12.5 pancreas with antibodies against Sox9 to mark progenitor cells and glucagon to mark differentiated cells. The size of the pancreatic epithelial bud and the number of glucagon cells from E12.5 embryos immunostained with Sox9 and glucagon were comparable in DNMT1 ${ }^{\mathrm{PC}}$ and control littermates (Fig. 2F-G). To further assess differentiation, we compared global gene expression by microarray analysis in control and DNMT1 $1^{\mathrm{PC}}$ pancreas. There was less than a twofold difference between most transcripts in the control and DNMT1 ${ }^{\text {PC }}$ pancreas, which suggests that the majority of the cells in the DNMT1 ${ }^{\mathrm{PC}}$ pancreas had a transcriptome similar to that of the control pancreas (Supplemental Fig. 3A). Gene enrichment analysis indicated that a subset of genes involved in pancreatic differentiation was specifically down-regulated. Neurogenin 3, the gene required for differentiation of all endocrine cell types, was one of the most down-regulated gene in the array analysis, with a $>12$-fold decrease in expression in the DNMT1 $1^{\text {PC }}$ 

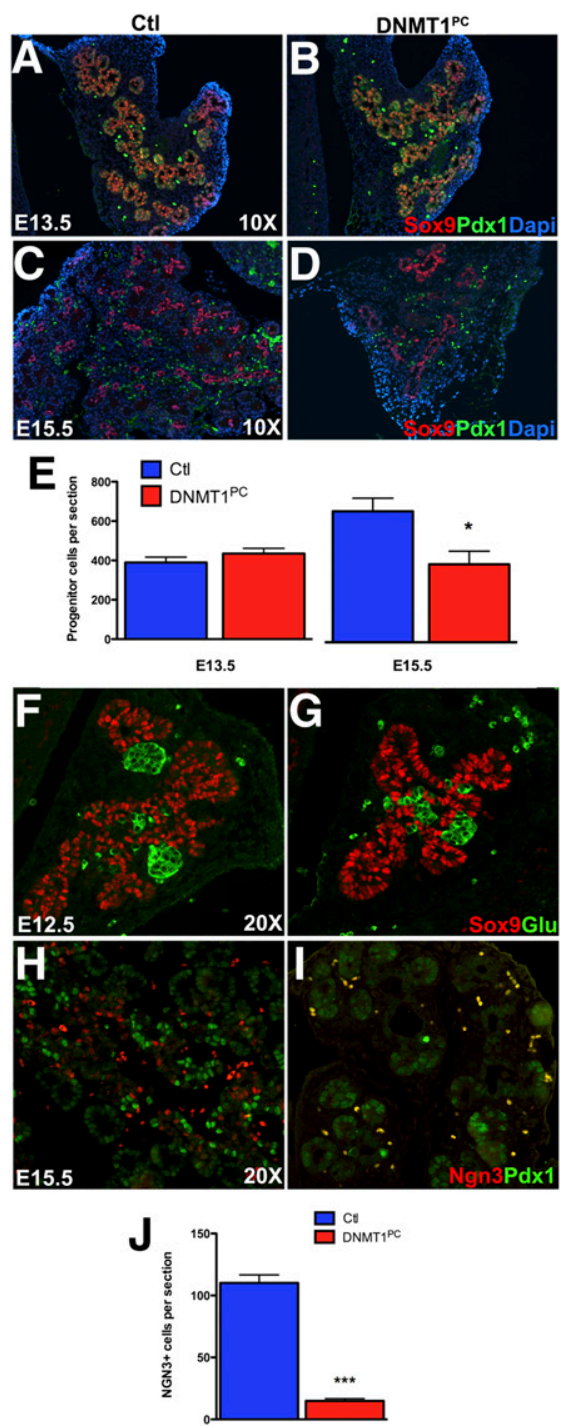

Figure 2. DNMT1 ${ }^{\mathrm{PC}}$ pancreatic atrophy is the result of progenitor cell depletion. $(A, B)$ Immunohistochemistry for Sox 9 and Pdx1 at E13.5 indicates no notable difference in the size or morphology in control $(A)$ and DNMT1 ${ }^{\mathrm{PC}}(B)$ pancreatic epithelium. $(C, D)$ Immunohistochemistry of pancreatic epithelium for Sox 9 and Pdx1 at E15.5 reveals a decrease in the number progenitor cells in the DNMT1 ${ }^{\mathrm{PC}}$ pancreatic epithelium. (E) Quantification of progenitor cells (E13.5) and Sox9 cells (E15.5). (F,G) Immunohistochemical comparison of the progenitor and glucagon populations at E12.5 in the control and DNMT1 ${ }^{\mathrm{PC}}$ suggests that precocious differentiation is not responsible for progenitor depletion. $(H, I)$. By E15.5, DNMT1 ${ }^{\text {PC }}$ pancreas have little expression of pre-endocrine cell marker Ngn3 $(I)$ in comparison with control littermates $(H)$. (J) Quantification of Ngn3 ${ }^{+}$cells at E15.5.

pancreas (Schwitzgebel et al. 2000). Other transcription factors involved in endocrine and exocrine lineage specification-such as Nkx6.1, Nkx2.2, $R f_{x} 6, R b j k-1$, and Ptf1-were specifically down-regulated (Supplemental Fig. 3B). We sought to confirm the gene expression profiles by immunohistochemistry. Immunostaining of the control pancreas at E15.5 showed $\mathrm{Ngn}^{+}$cells along the embryonic duct surrounded by pancreatic progenitor cells expressing $\mathrm{Pdx}$. In striking contrast, the number of $\mathrm{Ngn}_{3}{ }^{+}$cells in the DNMT1 $1^{\mathrm{PC}}$ pancreas was $<15 \%$ that of the control littermates $(P<0.005)$ (Fig. 2H-J). These results indicated that in the absence of Dnmt1, pancreatic epithelial cells were not depleted due to precocious differentiation, and in fact, very few differentiated cells were observed in the DNMT $1^{\mathrm{PC}}$ pancreas.

\section{Loss of Dnmt1 resulted in accumulation of progenitor} cells in the G2 phase

To investigate whether loss of Dnmt1 resulted in depletion of pancreatic progenitor cells due to defects in self-renewal, we analyzed embryos from pregnant dams injected with a short pulse of BrdU. Control and DNMT1 ${ }^{\text {PC }}$ pancreas were immunostained with antibodies against Pdx1 to mark pancreatic progenitor cells and BrdU to identify cells actively in $S$ phase of the cell cycle (Fig. 3A,B). The $\mathrm{Pdx} 1^{+} \mathrm{BrdU}^{+}$cells were counted to determine the proliferation index, calculated as $\left(\mathrm{Pdx} 1^{+} \mathrm{BrdU}^{+}\right) / \mathrm{Pdx}^{+}$. There were no differences in the proliferation index between control and DNMT1 $1^{\mathrm{PC}}$ progenitor cells at E12.5 (Fig. 3C). Because a short BrdU pulse only measures the cells that are actively in S phase, we pursued additional proliferation studies to determine whether the progenitor cells were completing the cell cycle. Control and DNMT1 ${ }^{\text {PC }}$ pancreas were immunostained with antibodies against phosphorylated histone H3, a marker for G2/M phase, and Pdx1 (Fig. 3D,E). Cells with a speckled $\mathrm{PHH} 3$ pattern were categorized as G2 phase of the cell cycle (Fig. 3D,E, white arrows), and cells with more robust staining were categorized as $M$ phase (Fig. 3D,E, orange arrows). Ratios of $\mathrm{Pdxl}^{+} \mathrm{PHH}^{+} / \mathrm{Pdxl}^{+}$ were used to calculate the proliferation index. Comparing control and DNMT1 ${ }^{\text {PC }}$ pancreas revealed that the number of M-phase progenitor cells was not significantly different, but the number of progenitor cells in G2 phase in the DNMT1 $1^{\mathrm{PC}}$ pancreas was increased by $40 \%(P<$ 0.005) (Fig 3F). The fact that S-phase and M-phase progenitor cells did not change in the DNMT1 ${ }^{\mathrm{PC}}$ pancreas indicated that cell cycle entry and mitosis of pancreatic epithelial cells were not perturbed by the deletion of Dnmt1. The accumulation of a subset of pancreatic
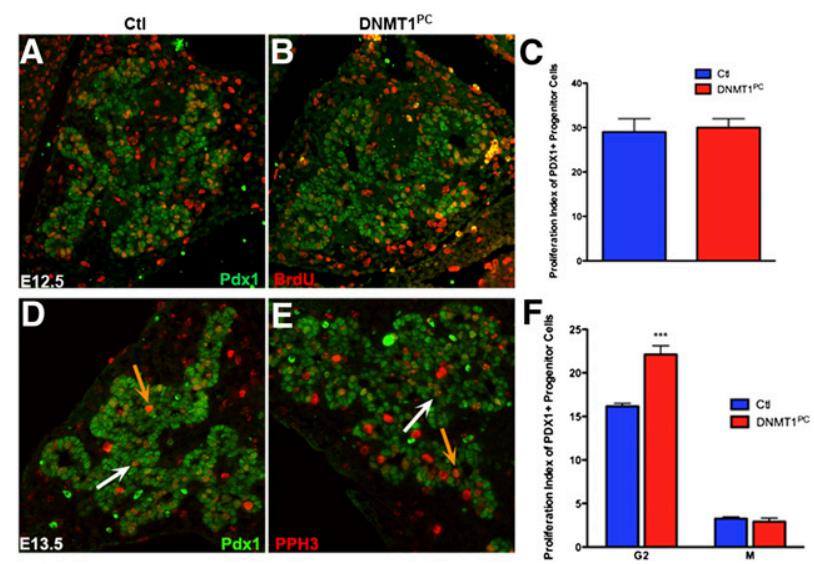

Figure 3. A subset of DNMT1 ${ }^{\mathrm{PC}}$ progenitor cells accumulates in G2 arrest. $(A-C)$ E12.5 pancreatic sections immunostained for BrdU and Pdx1 to quantify progenitor proliferation. $(D-F)$ Quantification of pancreatic progenitor cell proliferation at E13.5 as a percentage of phosphorylated histone $\mathrm{H}^{+} \mathrm{Pdxl}^{+}$double cells indicates that there is an increase in the number of cells in G2 in the DNMT1 ${ }^{\text {PC }}$ pancreas. (White arrows) G2-phase cells; (orange arrows) M-phase cells. $\left(^{\star \star \star}\right) P<0.005$. 
epithelial cells in G2 arrest suggested that depletion of these cells could be due to apoptosis.

\section{Deletion of Dnmt1 results in p53-dependent apoptosis of pancreatic progenitors}

We next investigated whether apoptosis of pancreatic epithelial cells could result in the atrophic phenotype of the DNMT1 ${ }^{\mathrm{PC}}$ pancreas at birth. Pancreatic sections from E15.5 DNMT1 ${ }^{\mathrm{PC}}$ and control littermate embryos were stained for cleaved caspase 3 , a marker for apoptosis, and Pdx1. Cleaved caspase 3 was not detected in control pancreas; however, DNMT1 ${ }^{\mathrm{PC}}$ pancreas showed a number of cells within the pancreatic epithelium that were positive for cleaved caspase 3 (Fig. 4A,B).

Several studies in fibroblast and cancer cell lines indicated that loss of Dnmt1 resulted in p53-dependent apoptosis, although the mechanism remains unclear (Jackson-Grusby et al. 2001; Chen et al. 2007; Anderson et al. 2009). To determine whether deletion of Dnmt1 in pancreatic progenitors resulted in changes in p53, we first assessed whether levels of p53 were altered in $\mathrm{DNMT} 1^{\mathrm{PC}}$ pancreas. Immunohistochemical analysis using a p53 antibody revealed that p53 accumulated in the DNMT1 ${ }^{\text {PC }}$ epithelium, while no p53 staining was
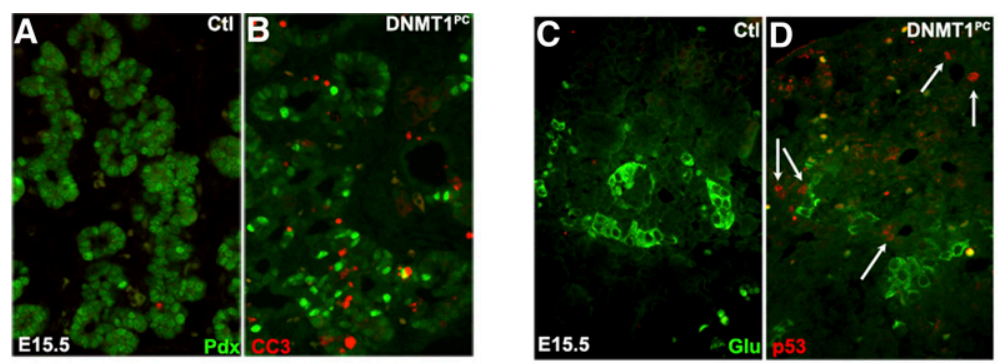

E
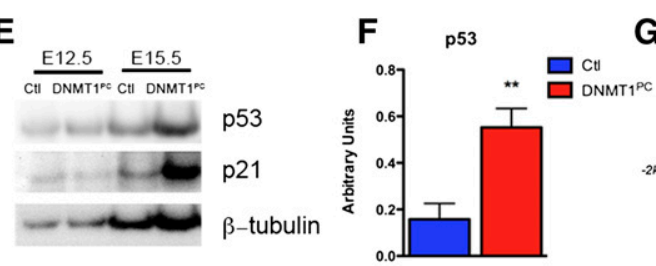

G
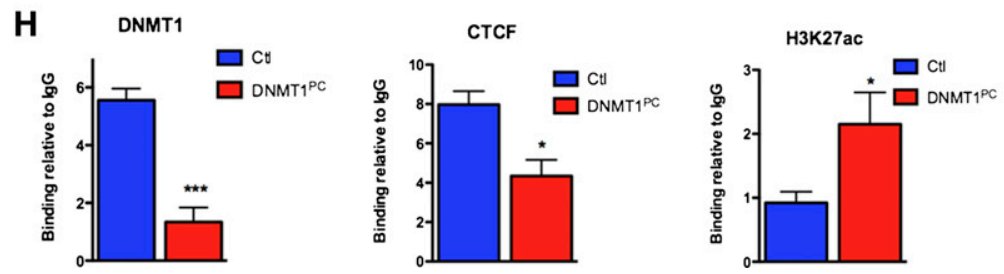

Figure 4. Progenitor depletion is a result of apoptosis in the DNMT1 ${ }^{\mathrm{PC}}$ pancreatic epithelium. $(A, B)$ Immunohistochemistry for cleaved caspase 3 did not reveal any apoptosis in the control pancreas $(A)$, but apoptosis was widespread in the DNMT1 ${ }^{\text {PC }}$ pancreas $(B) .(C, D)$ The presence of apoptosis markers coincides with the appearance of $\mathrm{p} 53$ accumulation in the DNMT1 ${ }^{\mathrm{PC}}$ pancreatic epithelium $(D$, white arrows) and is absent in control littermates at E15.5 $(C)$. (E) Western blot analysis for p53 protein and its downstream target, p21, at E12.5 and E15. $(F)$ Densitometric quantification of Western blots indicates a sixfold increase in p53 protein at E15.5. (G) Schematic of the ChIP assay binding in the p53 locus. $(H) \mathrm{ChIP}$ analysis indicates that DNMT1 binds to the p53 locus in control pancreas but is lost in $\mathrm{DNMT1}^{\mathrm{PC}}$ pancreas. Chromatin insulator protein CTCF is also bound to the p53 locus in control pancreas but is lost in the DNMT1 $1^{\text {PC }}$ pancreas. The activating histone mark $\mathrm{H} 3 \mathrm{~K} 27 \mathrm{ac}$ is enriched in the $\mathrm{DNMT1}{ }^{\mathrm{PC}}$ pancreas. observed in control littermate pancreas (Fig. 4C,D, white arrows). To confirm this accumulation of p53 in DNMT1 ${ }^{\mathrm{PC}}$ pancreas, we measured p53 using Western blot analysis at epithelium, and at E15.5, after the onset of p53 expresdifference in the accumulation of p53 at E12.5, but there was a significant sixfold increase in p53 in the DNMT1 ${ }^{\mathrm{PC}}$ mates at E15.5 $(P=0.0098)($ Fig 4E,F). Western blot analysis indicated that the p53 downstream transcriptional target $\mathrm{p} 21$ accumulated in the DNMT1 ${ }^{\mathrm{PC}}$ pancreas (Fig. 4E). To assess whether the increase in p53 protein used quantitative PCR to quantify any changes in p53 gene expression. Our analysis revealed a 20 -fold increase in p53 mRNA in DNMT1 $1^{\mathrm{PC}}$ pancreas compared with control pancreas from littermates (data not shown). The increase in p53 mRNA indicated that Dnmt1 could otentially regulate p53 at a transcriptional level. transcriptional up-regulation of $p 53$. We hypothesized that DNMT1 could play a role in directly repressing p53 transcription and that loss of Dnmt1 would result in derepression of $p 53$. Supporting our hypothesis, a previous study showed that DNMT1 was bound to the $p 53$ promoter $2 \mathrm{~kb}$ upstream of the $p 53$ transcription start site. Interestingly, this upstream regulatory region also contained a binding sequence for the transcriptional repressor CTCF. Binding of CTCF to this region suppressed reporter activity (Su et al. 2009). We reasoned that DNMT1 and CTCF associated at the $p 53$ regulatory region, and binding of CTCF to the $p 53$ regulatory region may be disrupted in the absence of Dnmt1. To test this, E14.5 pancreas from DNMT1 ${ }^{\text {PC }}$ and control littermates were subjected to chromatin immunoprecipitation (ChIP) analysis (Fig. 4G). In control pancreas, both DNMT1 and CTCF were bound to the $p 53$ regulatory region, confirming that DNMT1 and CTCF binding was associated with $p 53$ repression $(P<0.005)$ (Fig. $4 \mathrm{H})$. In contrast, there was no enrichment of CTCF in DNMT1 ${ }^{\text {PC }}$ embryos $(P=0.0268)$ (Fig. 4H). Moreover, enrichment of H3K27ac within the p53 promoter region was observed in the DNMT1 ${ }^{\text {PC }}$ embryos, which is consistent with transcriptional activation $(P=0.0423)$ (Fig. $4 \mathrm{H})$. These results suggested that DNMT1 directly repressed $p 53$ transcription, and loss of Dnmt 1 resulted in derepression of $p 53$.

\section{Haploinsufficiency of p53 rescues pancreatic organogenesis}

To investigate whether inactivation of Dnmt1 resulted in p53-mediated apoptosis of pancreatic progenitors, we crossed the p53-null animals into the DNMT1 ${ }^{\mathrm{PC}}$ background. p53-null animals are phenotypically indistinguishable from wild-type littermates at birth, and pancreas formation was not perturbed (Jacks et al. 1994).

We first assessed whether haploinsufficiency of $p 53$ restored pancreatic progenitor popula- 


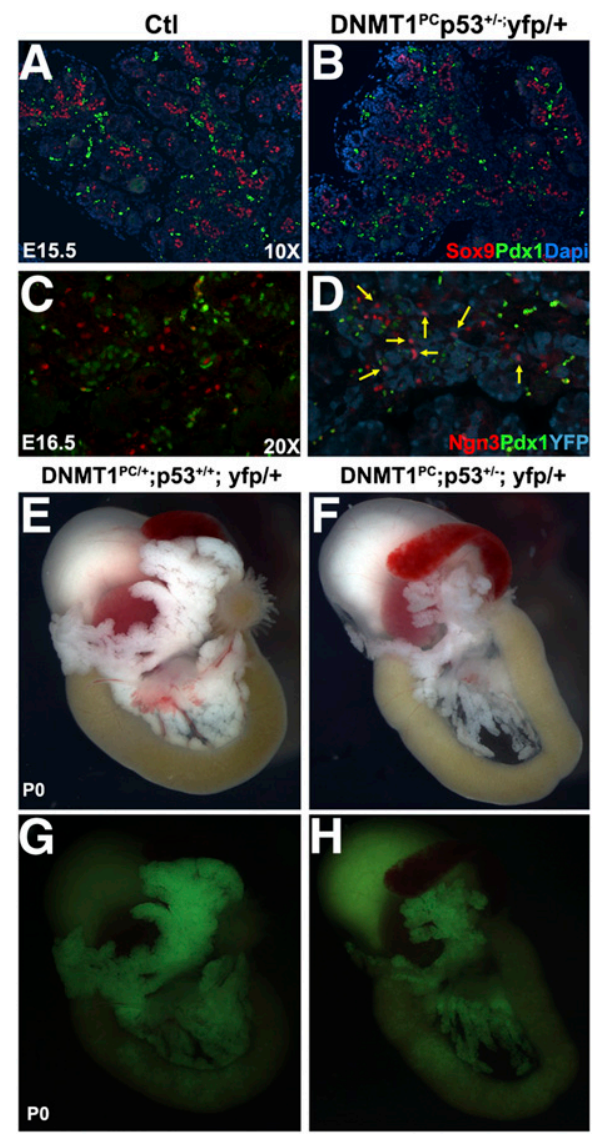

Figure 5. Haploinsufficiency of p53 rescues apoptosis and allows for cell survival during differentiation in $\mathrm{DNMT} 1^{\mathrm{PC}} \mathrm{p} 53^{+/-}$pancreas. $(A, B)$ Immunohistochemical staining for Sox 9 and Pdx indicates no significant difference in the control $(A)$ and DNMT1 ${ }^{\mathrm{PC}_{\mathrm{p}} 53^{+/-}}$ $(B)$ cell populations at E15.5. (C,D) Immunohistochemical analysis of

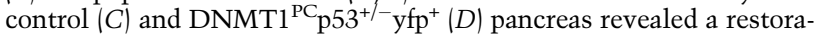
tion of $\mathrm{Ngn}^{+}$expression in the absence of Dnmt1. Overlap with YFP indicates that these cells have arisen from cells that underwent re-

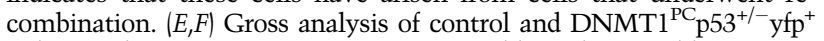
indicates that mutant pancreas are comparable with control littermates at PO. $(G, H)$ Lineage tracing by YFP expression indicates that most cells

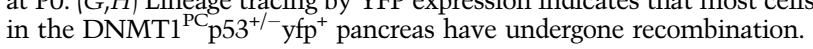

tions at E15.5. Immunohistochemical assessment of Sox9 and Pdx1 revealed no significant differences in the pancreatic epithelium between the control and DNMT1 ${ }^{\mathrm{PC}} \mathrm{p}_{53}{ }^{+/-}$pancreas (Fig. 5A,B). To evaluate whether $p 53$ haploinsufficiency would restore the expression of $n g n 3$, we examined DNMT $1^{\mathrm{PC}} \mathrm{p} 53^{+/-}$pancreas for expression of Ngn3. Immunohistochemistry at E16.5 indicated that $\mathrm{Ngn}^{+}$and $\mathrm{Pdxl}^{+}$cell populations persisted through organogenesis with a staining pattern similar to that of control littermates. Lineage tracing indicated that $\mathrm{DNMT} 1^{\mathrm{PC}} \mathrm{p} 53^{+/-}$cells expressing $\mathrm{Ngn} 3$ had undergone recombination, thus leading us to conclude that the expression of $\mathrm{Ngn} 3$ was restored by $p 53$ haploinsufficiency (Fig. 5C,D). At birth, DNMT1 ${ }^{\text {PC }}$ and DNMT $1^{\mathrm{PC}} \mathrm{p}_{53^{+/}}$animals were present at the expected Mendelian ratios. Gross histological examination of the neonatal pancreas revealed that while the pancreas of DNMT1 ${ }^{\text {PC }}$ animals were severely atrophic, DNMT1 ${ }^{\mathrm{PC}} \mathrm{p}_{5} 3^{+/-}$littermates had a restoration of a full, albeit smaller, pancreas (Fig. 5E,F). Lineage tracing suggested that recombination was extensive, although not complete, in the DNMT1 ${ }^{\mathrm{PC}} \mathrm{p} 53^{+/-}$background (Fig. 5G,H). Insulin and YFP immunostaining indicated normal morphology of the endocrine tissue compartment in the control and DNMT1 ${ }^{\mathrm{PC}} \mathrm{p} 3^{+/-}$littermate pancreas (Supplemental Fig. 4A,B). Immunostaining for amylase and YFP indicated that exocrine cells formed morphologically normal rosette structures (Supplemental Fig. 4C,D). $\mathrm{Mucin}^{+} / \mathrm{YFP}^{+}$ductal structures were present and morphologically normal in both the control and DNMT1 ${ }^{\mathrm{PC}} \mathrm{p}^{\mathrm{P}} 3^{+/-}$ pancreas (Supplemental Fig. 4E,F). Taken together, these data indicated that p53 haploinsufficiency rescued the ability of Dnmt1-null pancreatic progenitor cells to differentiate into a normal mature organ during embryogenesis. The area of DNMT1 ${ }^{\mathrm{PC}} \mathrm{p} 53^{+/-}$islets decreased by postnatal day 15 , and blood glucose levels were elevated into the diabetic range. This suggests that while haploinsufficiency of $p 53$ may permit organogenesis in the DNMT1 ${ }^{\mathrm{PC}}$ pancreas, these cells are not able to support glucose homeostasis in adolescent mice (Supplemental Fig. 5A-E).

Our results suggest that $p 53$ repression mediated by DNMT1 is not required for self-renewal of pancreatic progenitor cells per se but is critical for progenitor cell survival during differentiation. Furthermore, p53 haploinsufficiency prevented apoptosis and rescued the survival of Dnmt1-null progenitor cells; this suggests that p53 is a primary target of DNMT1 action in pancreatic progenitor cells. Recently, the role of Dnmt1 in epidermal progenitor cells suggested that Dnmt1 was required to retain proliferative stamina and suppress differentiation (Sen et al. 2010). Deletion of Dnmt1 in epidermal progenitors resulted in precocious differentiation and eventual tissue loss. Although the phenotype in the pancreatic deletions of Dnmt1 also was the eventual loss of tissue, no evidence of premature differentiation or defects in proliferation were observed. The mechanism by which Dnmt1 maintains progenitor populations may vary in different tissue depending on the cellular context. This suggests that at a molecular level, DNMT1 may associate with different effectors to carry out repressive functions depending on the cellular context.

We attribute the inability of the DNMT1 ${ }^{\mathrm{PC}}$ pancreatic progenitor population to undergo differentiation to the accumulation of p53. Part of p53 accumulation may be due to the derepression of the $p 53$ locus when DNMT1 is not present on the promoter. It is to be noted that there is no CpG methylation around the DNMT1-CTCFbinding area (Su et al. 2009; our unpublished results), suggesting that DNMT1 is working as part of a repressive complex independent of its DNA methylation activity. Methylation-independent functions of DNMT1 have been shown to repress target genes as part of complexes with E2F1 and histone deacetylases (Valdez et al. 2011; Clements et al. 2012). The inability of DNMT1 ${ }^{\text {PC }}$ cells to differentiate because of derepression of $p 53$ is similar to the $m d m 2^{-/-}$mouse phenotype (Jones et al. 1995; Montes de Oca Luna et al. 1995). While $m d m 2^{-1-}$ are embryonic-lethal at E6.5, $\mathrm{mdm} 2^{-1-} p 53^{-1-}$ compound mutants are normal and viable. Analysis of compound $\mathrm{mdm} 2^{-1-} \mathrm{p} 53^{515 \mathrm{c}-515 \mathrm{c}}$, which express a mutated p53 that can mediate growth arrest but not apoptosis, revealed hematapoetic and lymphoid deficiencies because of progenitor cell cycle and differentiation defects (Liu et al. 2007). Thus, it is possible that repression of p53 serves 
to facilitate pancreatic progenitor cell differentiation during normal organogenesis and that the absence of Dnmt1 results in transcriptional derepression and subsequent accumulation of $p 53$ that initiates cell cycle arrest and apoptosis during differentiation. The ability of DNMT1 ${ }^{\mathrm{PC}} \mathrm{p}_{5} 3^{+/-}$pancreatic progenitor cells to persist through differentiation and form a pancreas at birth suggests that Dnmt1 is not required to maintain progenitor cell self-renewal or differentiation but is required to attenuate the role of p53 as a checkpoint during differentiation.

\section{Materials and methods}

Information on mouse strains is in the Supplemental Material. All animal experiments were performed in accordance with NIH policies on the use of laboratory animals and approved by the Animal Research Committee of the Office for the Protection of Research Subjects at University of California at Los Angeles. For immunohistochemical experiments, tissues were prepared, oriented, and stained, and images were captured as previously described (Georgia et al. 2006). Calculations of the proliferative index were done as previously described using at least three embryos from each genotype (Zhong et al. 2007). ChIP experiments with the E14.5 dorsal pancreas cells were carried out using the micro-ChIP protocol (Dahl and Collas 2008). Statistical significance was determined using Student's $t$ test. Information on mouse strains, antibodies, and primers is in the Supplemental Material.

\section{Acknowledgments}

We thank Guoping Fan (University of California at Los Angeles) for providing the Dnmt $1^{\text {lox/lox }}$ mice and helpful comments. We are grateful to Lendy Le for technical assistance. S.G. is supported by an National Institute of Diabetes and Digestive and Kidney Diseases (NIDDK) K01 (DK088995). This work was supported by grants from NIDDK (DK080996 and DK068763), JDRF, and the Helmsley Trust to A.B. S.G. and M.K. performed the experiments. A.B. and S.G. conceived and planned the experiments and interpreted data. A.B. and S.G. wrote the manuscript.

\section{References}

Anderson RM, Bosch JA, Goll MG, Hesselson D, Dong PDS, Shin D, Chi $\mathrm{NC}$, Shin $\mathrm{CH}$, Schlegel A, Halpern M, et al. 2009. Loss of Dnmt1 catalytic activity reveals multiple roles for DNA methylation during pancreas development and regeneration. Dev Biol 334: 213-223.

Bhushan A, Itoh N, Kato S, Thiery JP, Czernichow P, Bellusci S, Scharfmann R. 2001. Fgf10 is essential for maintaining the proliferative capacity of epithelial progenitor cells during early pancreatic organogenesis. Development 128: 5109-5117.

Chen T, Hevi S, Gay F, Tsujimoto N, He T, Zhang B, Ueda Y, Li E. 2007. Complete inactivation of DNMT1 leads to mitotic catastrophe in human cancer cells. Nat Genet 39: 391-396.

Clements EG, Mohammad HP, Leadem BR, Easwaran H, Cai Y, Van Neste L, Baylin SB. 2012. DNMT1 modulates gene expression without its catalytic activity partially through its interactions with histone-modifying enzymes. Nucleic Acids Res 40: 4334-4346.

Dahl JA, Collas P. 2008. A rapid micro chromatin immunoprecipitation assay (microChIP). Nat Protoc 3: 1032-1045.

Fan G, Beard C, Chen RZ, Csankovszki G, Sun Y, Siniaia M, Biniszkiewicz D, Bates B, Lee PP, Kuhn R, et al. 2001. DNA hypomethylation perturbs the function and survival of CNS neurons in postnatal animals. J Neurosci 21: 788-797.

Fan G, Martinowich K, Chin MH, He F, Fouse SD, Hutnick L, Hattori D, $\mathrm{Ge} \mathrm{W}$, Shen $\mathrm{Y}, \mathrm{Wu} \mathrm{H}$, et al. 2005. DNA methylation controls the timing of astrogliogenesis through regulation of JAK-STAT signaling. Development 132: 3345-3356.

Georgia S, Soliz R, Li M, Zhang P, Bhushan A. 2006. p57 and Hes1 coordinate cell cycle exit with self-renewal of pancreatic progenitors. Dev Biol 298: 22-31.

Gittes GK. 2009. Developmental biology of the pancreas: A comprehensive review. Dev Biol 326: 4-35.
Gu G, Dubauskaite J, Melton DA. 2002. Direct evidence for the pancreatic lineage: $\mathrm{NGN3}^{+}$cells are islet progenitors and are distinct from duct progenitors. Development 129: 2447-2457.

Gu G, Brown JR, Melton DA. 2003. Direct lineage tracing reveals the ontogeny of pancreatic cell fates during mouse embryogenesis. Mech Dev 120: 35-43.

Herrera PL. 2000. Adult insulin- and glucagon-producing cells differentiate from two independent cell lineages. Development 127: 2317-2322.

Hutnick LK, Golshani P, Namihira M, Xue Z, Matynia A, Yang XW, Silva AJ, Schweizer FE, Fan G. 2009. DNA hypomethylation restricted to the murine forebrain induces cortical degeneration and impairs postnatal neuronal maturation. Hum Mol Genet 18: 2875-2888.

Jacks T, Remington L, Williams BO, Schmitt EM, Halachmi S, Bronson RT, Weinberg RA. 1994. Tumor spectrum analysis in p53-mutant mice. Curr Biol 4: 1-7.

Jackson-Grusby L, Beard C, Possemato R, Tudor M, Fambrough D, Csankovszki G, Dausman J, Lee P, Wilson C, Lander E, et al. 2001. Loss of genomic methylation causes p53-dependent apoptosis and epigenetic deregulation. Nat Genet 27: 31-39.

Jensen J. 2004. Gene regulatory factors in pancreatic development. DeV Dyn 229: 176-200.

Jensen J, Heller RS, Funder-Nielsen T, Pedersen EE, Lindsell C, Weinmaster G, Madsen OD, Serup P. 2000a. Independent development of pancreatic $\alpha$ - and $\beta$-cells from neurogenin3-expressing precursors: A role for the notch pathway in repression of premature differentiation. Diabetes 49: 163-176.

Jensen J, Pedersen EE, Galante P, Hald J, Heller RS, Ishibashi M, Kageyama R, Guillemot F, Serup P, Madsen OD. 2000b. Control of endodermal endocrine development by Hes-1. Nat Genet 24: 3644.

Jones SN, Roe AE, Donehower LA, Bradley A. 1995. Rescue of embryonic lethality in Mdm2-deficient mice by absence of p53. Nature 378: 206208.

Lei H, Oh SP, Okano M, Juttermann R, Goss KA, Jaenisch R, Li E. 1996. De novo DNA cytosine methyltransferase activities in mouse embryonic stem cells. Development 122: 3195-3205.

Li E, Bestor TH, Jaenisch R. 1992. Targeted mutation of the DNA methyltransferase gene results in embryonic lethality. Cell 69: 915-926.

Liu G, Terzian T, Xiong S, Van Pelt CS, Audiffred A, Box NF, Lozano G. 2007. The $\mathrm{p} 53-\mathrm{Mdm} 2$ network in progenitor cell expansion during mouse postnatal development. I Pathol 213: 360-368.

Montes de Oca Luna R, Wagner DS, Lozano G. 1995. Rescue of early embryonic lethality in mdm2-deficient mice by deletion of $\mathrm{p} 53$. Nature 378: 203-206.

Panning B, Jaenisch R. 1996. DNA hypomethylation can activate Xist expression and silence X-linked genes. Genes Dev 10: 1991-2002.

Reik, W. 2007. Stability and flexibility of epigenetic gene regulation in mammalian development. Nature 447: 425-432.

Schwitzgebel VM, Scheel DW, Conners JR, Kalamaras J, Lee JE, Anderson DJ, Sussel L, Johnson JD, German MS. 2000. Expression of neurogenin3 reveals an islet cell precursor population in the pancreas. Development 127: 3533-3542.

Sen GL, Reuter JA, Webster DE, Zhu L, Khavari PA. 2010. DNMT1 maintains progenitor function in self-renewing somatic tissue. Nature 463: 563-567.

Srinivas S, Watanabe T, Lin CS, William CM, Tanabe Y, Jessell TM, Costantini F. 2001. Cre reporter strains produced by targeted insertion of EYFP and ECFP into the ROSA26 locus. BMC Dev Biol 1: 4.

Su CH, Shann YJ, Hsu MT. 2009. p53 chromatin epigenetic domain organization and p53 transcription. Mol Cell Biol 29: 93-103.

Trowbridge JJ, Snow JW, Kim J, Orkin SH. 2009. DNA methyltransferase 1 is essential for and uniquely regulates hematopoietic stem and progenitor cells. Cell Stem Cell 5: 442-449.

Tschen S-I, Dhawan S, Gurlo T, Bhushan A. 2009. Age-dependent decline in $\beta$-cell proliferation restricts the capacity of $\beta$-cell regeneration in mice. Diabetes 58: 1312-1320.

Valdez CD, Davis JN, Odeh HM, Layfield TL, Cousineau CS, Berton TR, Johnson DG, Wojno KJ, Day ML. 2011. Repression of androgen receptor transcription through the E2F1/DNMT1 axis. PLOS ONE 6: e25187.

Zhong L, Georgia S, Tschen SI, Nakayama K, Bhushan A. 2007. Essential role of Skp2-mediated p27 degradation in growth and adaptive expansion of pancreatic $\beta$ cells. J Clin Invest 117: 2869-2876. 


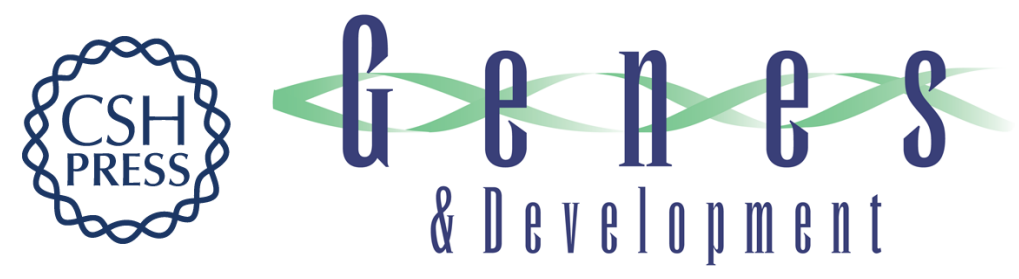

\section{DNMT1 represses $p 53$ to maintain progenitor cell survival during pancreatic organogenesis}

Senta Georgia, Murtaza Kanji and Anil Bhushan

Genes Dev. 2013, 27:

Access the most recent version at doi:10.1101/gad.207001.112

Supplemental
Material http://genesdev.cshlp.org/content/suppl/2013/02/21/27.4.372.DC1

References This article cites 33 articles, 10 of which can be accessed free at: http://genesdev.cshlp.org/content/27/4/372.full.html\#ref-list-1

License

Email Alerting

Receive free email alerts when new articles cite this article - sign up in the box at the top Service right corner of the article or click here.

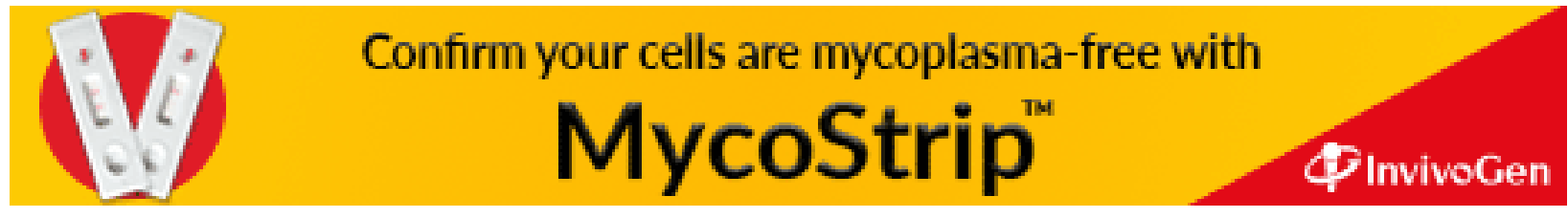

